\title{
Erratum to: Planted forest development in Australia and New Zealand: comparative trends and future opportunities
}

David Rhodes ${ }^{1 *}$ and Michael Stephens ${ }^{2}$

After publication of our work (Rhodes and Stephens 2014), we realised that incorrect data was included in Table five (Table 1 here). Specifically, most of the mean annual increment values and some of the rotation lengths were misquoted. The correct version of Table five (Table 1 here) is provided here.

Table 1 Australian regional growth rates for wood plantations

\begin{tabular}{|c|c|c|c|}
\hline Species & $\begin{array}{l}\text { Mean Annual } \\
\text { Increment } \\
\left(\mathrm{m}^{3} \mathrm{ha}^{-1} \text { year }^{-1}\right)\end{array}$ & $\begin{array}{l}\text { Rotation } \\
\text { length } \\
\text { (years) }\end{array}$ & Region(s) \\
\hline $\begin{array}{l}\text { Temperate } \\
\text { eucalypts } \\
\text { (pulpwood) }\end{array}$ & $16-25$ & $10-14$ & $\begin{array}{l}\text { Tasmania, South Australia, } \\
\text { New South Wales, Victoria, } \\
\text { Queensland, south-west } \\
\text { Western Australia }\end{array}$ \\
\hline $\begin{array}{l}\text { Temperate } \\
\text { eucalypts } \\
\text { (sawlog) }\end{array}$ & $13-20$ & $25-45$ & $\begin{array}{l}\text { Tasmania, South Australia, } \\
\text { New South Wales, Victoria, } \\
\text { Queensland, south-west } \\
\text { Western Australia }\end{array}$ \\
\hline Araucaria spp. & $13-15$ & 50 & $\begin{array}{l}\text { Queensland, northern } \\
\text { New South Wales }\end{array}$ \\
\hline Pinus radiata & $16-21$ & 30 & $\begin{array}{l}\text { Tasmania, Victoria, } \\
\text { New South Wales, } \\
\text { south-west Western } \\
\text { Australia, South Australia }\end{array}$ \\
\hline $\begin{array}{l}\text { Pinus elliotii and } \\
\text { P. caribaea hybrids }\end{array}$ & $12-18$ & 30 & $\begin{array}{l}\text { Queensland, northern } \\
\text { New South Wales }\end{array}$ \\
\hline Eucalyptus nitens & 15 & 15 & Tasmania \\
\hline
\end{tabular}

Source: Gavran et al. (2012).

\section{Author details}

${ }^{1}$ New Zealand Forest Owners Association, PO Box 10986, Wellington 6143 New Zealand. ${ }^{2}$ Australian Forest Products Association, PO Box 239, Deakin West ACT 2600, Australia.

Received: 25 December 2014 Accepted: 25 December 2014 Published online: 15 April 2015

\section{References}

Gavran, M, Frakes, I, Davey, S, \& Mahendrarajah, S. (2012). Australia's plantation log supply 2010-2054. Canberra: Australian Bureau of Agricultural and Resource Economics and Sciences.

Rhodes, D, \& Stephens, M. (2014). Planted forest development in Australia and New Zealand: comparative trends and future opportunities. New Zealand Journal of Forestry Science, 44(Suppl 1), S10. http://www.nzjforestryscience. com/content/44/S1/S10

\section{Submit your manuscript to a SpringerOpen ${ }^{\circ}$ journal and benefit from: \\ - Convenient online submission \\ - Rigorous peer review \\ - Immediate publication on acceptance \\ - Open access: articles freely available online \\ - High visibility within the field \\ - Retaining the copyright to your article}

Submit your next manuscript at $\boldsymbol{\wedge}$ springeropen.com

\footnotetext{
*Correspondence: david.rhodes@nzfoa.org.nz

${ }^{1}$ New Zealand Forest Owners Association, PO Box 10986, Wellington 6143 New Zealand

Full list of author information is available at the end of the article
}

\section{实}

(c) 2015 Rhodes and Stephens; licensee Springer. This is an Open Access article distributed under the terms of the Creative Commons Attribution License (http://creativecommons.org/licenses/by/4.0), which permits unrestricted use, distribution, and reproduction in any medium, provided the original work is properly credited. 